\title{
Asymptotic analysis of Lorentzian spin foam models
}

\author{
John W. Barrett \\ School of Mathematical Sciences, University of Nottingham \\ University Park, Nottingham NG7 2RD, UK \\ E-mail: john.barrett@nottingham.ac.uk
}

\section{R. J. Dowdall}

School of Physics \& Astronomy

Kelvin Building, University of Glasgow, Glasgow G12 8QQ, UK

E-mail: r.dowdall@physics.gla.ac.uk

Winston J. Fairbairn*

Department of Mathematics, FAU Erlangen-Nürnberg

Cauerstrasse 11, 91054 Erlangen, Germany

E-mail: winston.fairbairn@math.uni-erlangen.de

Frank Hellmann

Albert Einstein Institute

Mühlenberg 1, 14476 Golm, Germany

E-mail: hellmann@aei.mpg.de

\section{Roberto Pereira}

Instituto de Cosmologia Relatividade e Astrofisica ICRA - CBPF,

Rua Dr. Xavier Sigaud 150, CEP 22290-180, Rio de Janeiro, Brazil

E-mail: pereiraecbpf.br

We analyse the asymptotic properties of the amplitude for the 4-simplexes of the Lorentzian EPRL model using stationary phase methods. We compute the critical point equations and study the geometry of their solutions. For boundary data corresponding to a Lorentzian simplex, the asymptotic formula has two terms, with phase plus or minus the Lorentzian signature Regge action for the 4-simplex geometry, multiplied by an Immirzi parameter.

3rd Quantum Gravity and Quantum Geometry School

February 28 - March 13, 2011

Zakopane, Poland

\footnotetext{
* Speaker.
} 


\section{Introduction}

Spin foam models originated in the study of three-dimensional quantum gravity with the Ponzano-Regge model [1]. The relation between this model and gravity was first understood through the study of its asymptotics; the large spin behaviour of the $6 j$ symbol is a simple function of the Regge action for three-dimensional gravity. Modern research in quantum gravity attempts to generalise the Ponzano-Regge construction to higher dimensions.

A spin foam model in $n$ dimensions is based on a triangulation of the space-time manifold and is constructed by assigning (quantum) group representation theory data to the simplexes of the triangulation of dimension $(n-2)$ and $(n-1)$. A number is constructed from this data and is associated to the higher dimensional simplexes of the triangulation. This number is called an amplitude.

The interpretation of such models as quantum gravity models relies on their ability to reproduce general relativity in the low energy, semi-classical regime. A key step in the semi-classical analysis of these models is the study of the asymptotic behaviour of the amplitude for the $n$ simplexes.

In this paper, we present a study of the asymptotics of the amplitude for the 4-simplexes of the so-called EPRL model [2] for four-dimensional manifolds of Lorentzian signature. This presentation is based on the paper [3] and we refer the interested reader to the original article for further details.

\section{Four-simplex amplitude}

Let $M$ be a triangulated, closed, oriented four-dimensional manifold and $\sigma$ be a 4-simplex of $M$. The amplitude associated to $\sigma$ is determined by data associated to its boundary $\partial \sigma$.

\subsection{Boundary state space}

We first introduce some notation. Let the spin $k \in \mathbb{N} / 2$ label the unitary, irreducible representations $\pi_{k}: \mathrm{SU}(2) \rightarrow V_{k}$ of the Lie group SU(2). The tetrahedra $t$ of $\sigma$, all assumed to be space-like, will be labeled by latin indices $a, b=1, \ldots, 5$ which implies that couples of indices $(a b), a \neq b$, will refer to the triangles of $\sigma$.

Given the assignment

$$
k: \text { triangles } \rightarrow \operatorname{Irrep}(\mathrm{SU}(2)),
$$

of a spin $k_{a b}$ to each triangle of $\partial \sigma$, one can associate a vector space to each tetrahedron $t$ of $\partial \sigma$ called a state space

$$
\mathscr{H}_{a}=\operatorname{Inv}_{\mathrm{SU}(2)}\left(\bigotimes_{b: b \neq a} V_{k_{a b}}\right) \cong \operatorname{Hom}_{\mathrm{SU}(2)}\left(\mathbb{C}, \bigotimes_{b: b \neq a} V_{k_{a b}}\right)
$$

The direct sum over all possible spin assignments of the vector space $\mathscr{H}_{a}$ is the Hilbert space of quantum states of the $a$-th tetrahedron $[4,5]$. In this interpretation, the vector space $V_{k}$ is viewed as the Hilbert space of quantised bivectors of area $k$. 
The amplitude for the four-simplex $\sigma$ is a linear map from the tensor product $\mathscr{H}_{\partial \sigma}$ of the state spaces associated to the tetrahedra of $\partial \sigma$ to the complex numbers

$$
A_{\sigma}: \mathscr{H}_{\partial \sigma} \rightarrow \mathbb{C}, \quad \mathscr{H}_{\partial \sigma}=\bigotimes_{a} \mathscr{H}_{a} .
$$

A key step towards posing the asymptotic problem is to parametrise each $\mathscr{H}_{a}, a=1, \ldots, 5$, using coherent states [6]. A coherent state for the direction $\mathbf{n} \in S^{2} \subset \mathbb{R}^{3}$ and spin $k$ is a unit vector $\xi$ in $V_{k}$ defined, up to a phase, by the condition $(\mathbf{J} \cdot \mathbf{n}) \xi=i k \xi$, where $\mathbf{J}$ is a three-vector whose components are the standard anti-Hermitian generators of $\mathfrak{s u}(2)$ and the dot '. ' is the 3d Euclidean inner product. In the coherent state basis, the state $\Psi_{a} \in \mathscr{H}_{a}$ associated to the tetrahedron $a$ is given by assigning coherent states to the four boundary triangles $a b$, with fixed $a$ and varying $b$, and by $\mathrm{SU}(2)$-averaging the four-fold tensor product using the Haar measure on $\mathrm{SU}(2)$

$$
\Psi_{a}(k, \mathbf{n})=\int_{\mathrm{SU}(2)} d h \bigotimes_{b: b \neq a} \pi_{k_{a b}}(h) \xi_{a b}
$$

The boundary state for the boundary of $\sigma$ is

$$
\Psi(k, \mathbf{n})=\bigotimes_{a} \Psi_{a} \in \mathscr{H}_{\partial \sigma} .
$$

The data $\left\{k_{a b}, \mathbf{n}_{a b}\right\}$ specifying the boundary state up to a phase is called the boundary data. The asymptotic formula depends on this boundary data and certain classes of boundary data will play a paramount role in the following.

A boundary data is called non-degenerate if, for each tetrahedron $a$, the face vectors $\mathbf{n}_{a b}$ corresponding to the coherent states $\xi_{a b}$ for fixed $a$ and varying $b$ span a three-dimensional space. In this case, if the four vectors $\mathbf{n}_{a b}$ satisfy the closure condition

$$
\sum_{b: b \neq a} k_{a b} \mathbf{n}_{a b}=0
$$

they specify an embedding of the tetrahedron in three-dimensional Euclidean space, such that the vectors are the outward face normals and the $k_{a b}$ are the areas. In this way, each tetrahedron inherits a metric and an orientation but the metrics and orientations of different tetrahedra do not necessarily match. Non-degenerate boundary data for the whole 4-simplex is said to be geometric or Regge-like if the individual tetrahedron metrics and orientations glue together consistently to form an oriented Regge-calculus positive definite 3-metric for the boundary of the 4-simplex. This is the requirement that the induced metrics on the triangles agree for both of the tetrahedra sharing any given triangle, and the induced orientations are opposite.

For geometric boundaries, one can make a canonical choice of phase for the boundary state. For this type of boundary data, there exists a unique ${ }^{1}$ set of ten SU(2) elements $g_{a b}=g_{b a}^{-1}$ which glue together the oriented geometric tetrahedra of the boundary and map the outward normal to one tetrahedron to the inward normal to the other $g_{b a} \mathbf{n}_{a b}=-\mathbf{n}_{b a}$. From this data, one can select the phases of the coherent states by the condition

$$
\xi_{b a}=g_{b a} J \xi_{a b},
$$

where $J: V_{k} \rightarrow V_{k}$ is the $\mathrm{SU}(2)$ anti-linear structure map associated to the representation $k$. The boundary state $\Psi$ with this choice of phase is called a geometric state.

\footnotetext{
${ }^{1}$ Strictly speaking, this is true only up to a $\mathbb{Z}_{2}$ lift ambiguity discussed in [7].
} 


\subsection{Amplitude}

The Lorentzian EPRL model is based on the representation theory of the cover of the Lorentz group, the real Lie group $\operatorname{SL}(2, \mathbb{C})_{\mathbb{R}}$. More precisely, we will work with the unitary representations of the so-called principal series. These representations, labeled by a couple of parameters $\alpha=$ $(n, p)$, where $n \in \mathbb{Z} / 2$ and $p \in \mathbb{R}$, act in the Hilbert space $V_{\alpha}$ of functions on $\mathbb{C}^{2}$ satisfying the homogeneity property

$$
f(\lambda z)=\lambda^{-1+i p+k} \bar{\lambda}^{-1+i p-k} f(z), \quad z=\left(z_{0}, z_{1}\right) \in \mathbb{C}^{2} .
$$

The inner product on $V_{\alpha}$ is given by the expression

$$
\forall f, g \in V_{\alpha}, \quad(f, g)=\int_{\mathbb{C} P^{1}} \Omega \bar{f} g,
$$

where $\Omega$ is the standard invariant two-form on $\mathbb{C}^{2}-\{0\}$. Note that the integration range is the complex projective line because the combination $\Omega \bar{f} g$ is a two-form on $\mathbb{C} P^{1}$, i.e., has the right homogeneity to project down from $\mathbb{C}^{2}-\{0\}$ to $\mathbb{C} P^{1}$.

The principal representations factorise into representations of a $\mathrm{SU}(2)$ subgroup as follows

$$
V_{\alpha}=\bigoplus_{j=|n|}^{\infty} V_{j} .
$$

The Lorentzian EPRL construction [2] is based on a map injecting the boundary SU(2) representations into principal representations of the Lorentz group. This map depends on a fixed real parameter $\gamma \in \mathbb{R}^{+}$, called the Immirzi parameter, and is given by

$$
\begin{aligned}
f_{\gamma}: \operatorname{Irrep}(\mathrm{SU}(2)) & \rightarrow \operatorname{Irrep}\left(\operatorname{SL}(2, \mathbb{C})_{\mathbb{R}}\right) \\
k & \mapsto \alpha(k)=(n(k), p(k)):=(k, \gamma k) .
\end{aligned}
$$

We see that, in particular, the boundary $\mathrm{SU}(2)$ representation $k$ is identified with the lowest $\mathrm{SU}(2)$ subgroup factor of $\alpha=(n, p)$, that is, $k=n$. This identification leads to an embedding of the coherent states for the boundary tetrahedra into the vector space of intertwiners for the Lorentz group. Using the graphical calculus introduced in [3], the obtained intertwiners can be paired together according to the combinatorics of the complete graph with five vertices to yield a number. This number, given by the equation below, is the amplitude for the 4-simplexes

$$
A_{\sigma}(k, \mathbf{n})=(-1)^{\chi} \int_{\left(\operatorname{SL}(2, \mathbb{C})_{\mathbb{R}}\right)^{5}} \prod_{a} d X_{a} \delta\left(X_{5}\right) \prod_{a<b} P_{a b} .
$$

Here, the delta function fixes the non-compact $\operatorname{SL}(2, \mathbb{C})_{\mathbb{R}}$ symmetry of the amplitude and $(-1)^{\chi}$ is a sign determined by the graphical calculus. The kernel $P_{a b}$ is defined by

$$
P_{a b}=c_{a b} \int_{\mathbb{C} P^{1}} \Omega\left\langle X_{a}^{\dagger} z, X_{a}^{\dagger} z\right\rangle^{-1-i p_{a b}-n_{a b}}\left\langle X_{a}^{\dagger} z, \xi_{a b}\right\rangle^{2 n_{a b}}\left\langle X_{b}^{\dagger} z, X_{b}^{\dagger} z\right\rangle^{-1+i p_{a b}-n_{a b}}\left\langle J \xi_{b a}, X_{b}^{\dagger} z\right\rangle^{2 n_{a b}} .
$$

Here, $\xi \in \mathbb{C}^{2}$ is a coherent state in the fundamental $k=1 / 2$ representation, $X^{\dagger}$ is the Hermitian conjugate of $X$, and $\langle$,$\rangle is the Hermitian inner product on \mathbb{C}^{2}$. The $(n, p)$ are constrained by equation (2.10) to be of the form $(n, p)=(k, \gamma k)$ and $c_{a b}$ is a constant given explicitly in [3]. 


\section{Asymptotic analysis}

The goal of this paper is to study the asymptotics of the amplitude for the 4-simplexes when the spins $k$ grow large. This amounts to looking at the semi-classical regime of the model. Indeed, in [2] a formula for the area of the triangle $(a b)$ is given

$$
\operatorname{Area}(a b) \sim 8 \pi G \gamma \hbar k_{a b}
$$

The semi-classical regime consists in taking the limit $\hbar \rightarrow 0$. Since the physical area is kept fixed, this amounts to considering the limit $k \rightarrow \infty$.

To study this asymptotic problem, we will rescale all spins homogeneously $k_{a b} \mapsto \lambda k_{a b}$ and consider the regime where $\lambda \gg 1$.

\subsection{Asymptotic problem and critical points}

The first step in the study of the asymptotics is to put the integral expression for the 4-simplex amplitude (2.11) in an exponential form as a means to use stationary phase methods. In the following, the combinations $Z_{a b}=X_{a}^{\dagger} z_{a b}$ and $Z_{b a}=X_{b}^{\dagger} z_{a b}$, for each $a<b$ occur frequently; this notation will be used as a shorthand.

Using this notation, the kernel (2.12) can be written as

$$
P_{a b}=c_{a b} \int_{\mathbb{C} P^{1}} \Omega_{a b}\left(\frac{\left\langle Z_{b a}, Z_{b a}\right\rangle}{\left\langle Z_{a b}, Z_{a b}\right\rangle}\right)^{i p_{a b}}\left(\frac{\left\langle Z_{a b}, \xi_{a b}\right\rangle\left\langle J \xi_{b a}, Z_{b a}\right\rangle}{\left\langle Z_{a b}, Z_{a b}\right\rangle^{1 / 2}\left\langle Z_{b a}, Z_{b a}\right\rangle^{1 / 2}}\right)^{2 n_{a b}},
$$

where

$$
\Omega_{a b}=\frac{\Omega}{\left\langle Z_{a b}, Z_{a b}\right\rangle\left\langle Z_{b a}, Z_{b a}\right\rangle},
$$

which is a measure on $\mathbb{C} P^{1}$. Therefore, the rescaled four-simplex amplitude can be re-expressed as follows

$$
A_{\sigma}(\lambda k, \mathbf{n})=(-1)^{\chi} \int_{\left(\mathrm{SL}(2, \mathbb{C})_{\mathbb{R}}\right)^{5}} \prod_{a} d X_{a} \delta\left(X_{5}\right) \int_{\left(\mathbb{C} P^{1}\right)^{10}} \prod_{a<b} c_{a b} \Omega_{a b} \exp \left(\lambda S_{k, \mathbf{n}}[X, z]\right),
$$

with the action $S$ for the stationary problem given by

$$
S_{k, \mathbf{n}}[X, z]=\sum_{a<b} n_{a b} \ln \frac{\left\langle Z_{a b}, \xi_{a b}\right\rangle^{2}\left\langle J \xi_{b a}, Z_{b a}\right\rangle^{2}}{\left\langle Z_{a b}, Z_{a b}\right\rangle\left\langle Z_{b a}, Z_{b a}\right\rangle}+i p_{a b} \ln \frac{\left\langle Z_{b a}, Z_{b a}\right\rangle}{\left\langle Z_{a b}, Z_{a b}\right\rangle},
$$

where the couple $(n, p)$ is constrained according to (2.10). Note that the first term of the action is complex and the second term is purely imaginary.

We are now in a framework where stationary phase methods can apply. The asymptotic formula is dominated by the critical points of $S$, that is, the stationary points for which $\operatorname{Re} S$, the real part of $S$, is a maximum. We first investigate the condition on the real part of the action before looking at the stationary points. 
Condition on the real part of the action. From the expression of the real part of the action

$$
\operatorname{Re} S_{k, \mathbf{n}}[X, z]=\sum_{a<b} k_{a b} \ln \frac{\left|\left\langle Z_{a b}, \xi_{a b}\right\rangle\right|^{2}\left|\left\langle J \xi_{b a}, Z_{b a}\right\rangle\right|^{2}}{\left\langle Z_{a b}, Z_{a b}\right\rangle\left\langle Z_{b a}, Z_{b a}\right\rangle}
$$

one can deduce that it satisfies $\operatorname{Re} S \leq 0$ and is hence a maximum where it vanishes. The maximality requirement leads to the first set of critical point equations

$$
\forall a<b, \quad\left(X_{a}^{\dagger}\right)^{-1} \xi_{a b}=\frac{\left\|Z_{b a}\right\|}{\left\|Z_{a b}\right\|} e^{i \theta_{a b}}\left(X_{b}^{\dagger}\right)^{-1} J \xi_{b a},
$$

where $\theta_{a b}$ is a phase and $\|Z\|^{2}=\langle Z, Z\rangle$. We now turn towards the variational problem of the action.

Stationary points. The strategy is here to evaluate the first variation of the action on the solutions to equation (3.2). The variation with respect to the spinor variables $z_{a b}$ evaluated on the points maximising the real part of the action yields the second set of critical point equations

$$
\forall a<b, \quad X_{a} \xi_{a b}=\frac{\left\|Z_{a b}\right\|}{\left\|Z_{b a}\right\|} e^{i \theta_{a b}} X_{b} J \xi_{b a} .
$$

The variation with respect to the variables $\bar{z}_{a b}$ leads to the complex conjugate equation.

Finally, we compute the variation of the action with respect to the group variables. The right variation of an arbitrary $\operatorname{SL}(2, \mathbb{C})$ element $X$ and its Hermitian conjugate are given by

$$
\delta X=X \circ L, \quad \text { and } \delta X^{\dagger}=L^{\dagger} \circ X^{\dagger},
$$

where $L$ is an arbitrary element of $\mathfrak{s l}(2, \mathbb{C})_{\mathbb{R}}$. Using the vector space isomorphism

$$
\mathfrak{s l}(2, \mathbb{C})_{\mathbb{R}}=\left(\mathfrak{s u}(2)^{\mathbb{C}}\right)_{\mathbb{R}} \cong \mathfrak{s u}(2) \oplus i \mathfrak{s u}(2),
$$

we can decompose $L$ into a rotational and boost part $L=\alpha^{i} J_{i}+\beta^{i} K_{i}$, with $\alpha^{i}, \beta^{i}$ in $\mathbb{R}$ for all $i=1,2,3$. Using the above and the fact that the spinors $\xi_{a b}$ determine $\mathrm{SU}(2)$ coherent states,

$$
\left\langle\xi_{a b}, \mathbf{J} \xi_{a b}\right\rangle=\frac{i}{2} \mathbf{n}_{a b}, \quad \text { and } \quad\left\langle\xi_{a b}, \mathbf{K} \xi_{a b}\right\rangle=\frac{1}{2} \mathbf{n}_{a b},
$$

we can evaluate the variation of the action on the solutions to (3.2). As a result, we obtain the last set of critical point equations

$$
\sum_{b: b \neq a} k_{a b} \mathbf{n}_{a b}=0 .
$$

There is one such vector equation for each tetrahedron $a$.

\subsection{Geometry of the critical points}

Here, we make the assumption that the boundary data is such that the critical point equations (3.2), (3.3) admit a non-trivial solution $\left\{X_{a}\right\}$. The idea to extract the geometry of the critical points is to use the bivector geometry theorem proved in $[8,9]$. This theorem states, in particular, what constraints an abstract set of ten bivectors should satisfy to determine a geometric 4-simplex via an identification to the geometric bivectors associated to the triangles of the simplex. The first step 
towards the construction of such bivectors is to extract Minkowski vectors from the asymptotic data. This is based on the identification between spinors and null vectors.

Let $\gamma: \mathbb{R}^{3,1} \rightarrow \mathbb{H}$ be the standard isomorphism between Minkowski space $\mathbb{R}^{3,1}$ and the space of $2 \times 2$ hermitian matrices $\mathbb{H}$. Call $\mathbb{H}_{0}^{+}$the subset defined by

$$
\mathbb{H}_{0}^{+}=\{h \in \mathbb{H} \mid \operatorname{det} h=0, \text { and } \operatorname{Tr} h>0\} .
$$

The isomorphism $\gamma$ identifies the future null cone $C^{+}$in Minkowski space with $\mathbb{H}_{0}^{+}$because $\operatorname{det} \gamma(x)=-\eta(x, x)$, where $\eta$ is the flat Minkowski metric with signature -+++ . Therefore, using the standard map between spinors and elements of $\mathbb{H}_{0}^{+}$

$$
\zeta: \mathbb{C}^{2} \rightarrow \mathbb{H}_{0}^{+}, \quad z \mapsto \zeta(z)=z \otimes z^{\dagger},
$$

one can define a map $\imath: \mathbb{C}^{2} \rightarrow C^{+} \subset \mathbb{R}^{3,1}$. Following this construction, one can associate two null vectors

$$
\imath(\xi)=\frac{1}{2}(1, \mathbf{n}), \quad \text { and } \quad \imath(J \xi)=\frac{1}{2}(1,-\mathbf{n}),
$$

to a given coherent state $\xi$.

From these two vectors, one can construct the space-like bivector

$$
b=2 * \imath(J \xi) \wedge \imath(\xi),
$$

where the star $*$ is the Hodge operator on $\Lambda^{2}\left(\mathbb{R}^{3,1}\right)$. Regarded as an anti-symmetric $4 \times 4$ matrix, $b$ is given explicitly by

$$
b=*\left[\begin{array}{cccc}
0 & n^{1} & n^{2} & n^{3} \\
-n^{1} & 0 & 0 & 0 \\
-n^{2} & 0 & 0 & 0 \\
-n^{3} & 0 & 0 & 0
\end{array}\right] .
$$

Thus, to every coherent state $\xi_{a b}$ of the asymptotic data, one can associate a space-like bivector $b_{a b}$. In fact, the critical point equations (3.2), (3.3) carry a richer geometric structure. This geometry is made transparent by acting with a Lorentz transformation on the bivectors $b_{a b}$ and defining the space-like bivectors

$$
B_{a b}=k_{a b} \hat{X}_{a} \otimes \hat{X}_{a} b_{a b}
$$

where $\hat{X}_{a}$ is the $\operatorname{SO}(3,1)$ element corresponding to $\pm X_{a}$ in $\operatorname{SL}(2, \mathbb{C})_{\mathbb{R}}$.

These bivectors satisfy the bivector geometry conditions $[8,9]$. They are simple and crosssimple by construction. The normal appearing in the cross-simplicity condition for tetrahedron $a$ is here a future pointing vector $F_{a}$ in the future hyperboloid $H_{3}^{+}$. This vector is the image of the reference vector $\mathscr{F}=(1,0,0,0) \in H_{3}^{+}$under the action of $X_{a}$, that is, $\gamma\left(F_{a}\right)=X_{a} X_{a}^{\dagger}$. Furthermore, the constructed bivectors satisfy closure and orientation because of the closure condition (3.6) and the spinor equations (3.2), (3.3) satisfied by the critical points. To show the orientation equation, one uses the action of $J$ on $\operatorname{SL}(2, \mathbb{C})$ given by $J X J^{-1}=\left(X^{\dagger}\right)^{-1}$, for all $X$ in $\operatorname{SL}(2, \mathbb{C})$. Under the assumption of non-degeneracy of the boundary data, these bivectors also satisfy a tetrahedron condition. Finally, the critical points determine normals $F_{a}$ which are either such that at least three out of the five normals are linearly independent, or such that all are pointing in the same direction. 
In the first case, which occurs when the solution $\left\{X_{a}\right\}$ does not lie in the $\mathrm{SU}(2)$ subgroup stabilising $\mathscr{F}$, the corresponding bivectors satisfy the non-degeneracy condition of a Minkowskian bivector geometry and all bivector geometry conditions are fulfilled.

This implies that we have an equivalence between non-degenerate solutions to the critical point equations and geometric 4-simplexes up to inversion. In particular, for every solution that is non-degenerate, there exists a parameter $\mu$ which takes the value either 1 or -1 , and an inversionrelated pair of Lorentzian 4-simplexes $\sigma$. These are such that the bivectors (of either of the two simplexes) $B_{a b}(\sigma)$ satisfy

$$
B_{a b}(\sigma)=\mu k_{a b} \hat{X}_{a} \otimes \hat{X}_{a} b_{a b}
$$

\section{Asymptotic formula}

In this section, we make the assumption that the boundary data is that of a geometric Lorentzian 4-simplex. In this case, there are two distinct solutions to the critical point equations related by a parity transformation [3]. We evaluate the action on such solutions and give the asymptotic formula.

\subsection{Regge action}

The action at the critical points has vanishing real part and we are left with the imaginary part

$$
S_{k, \mathbf{n}}=i \sum_{a<b} p_{a b} \ln \frac{\left\|Z_{b a}\right\|^{2}}{\left\|Z_{a b}\right\|^{2}}+2 n_{a b} \theta_{a b}
$$

In the case considered here where the critical points determine a non-degenerate 4-simplex with Lorentzian metric, the logarithms are related to the dihedral angles of the simplex. To understand this point, consider a solution to the critical point equations and couple the two spinor equations (3.2), (3.3). The result is the eigenvalue equation

$$
L_{a b} \xi_{a b}=e^{r_{a b}} \xi_{a b}, \quad \text { with } L_{a b}=X_{a}^{-1} X_{b} X_{b}^{\dagger}\left(X_{a}^{\dagger}\right)^{-1},
$$

and $e^{r_{a b}}=\left\|Z_{b a}\right\|^{2} /\left\|Z_{a b}\right\|^{2}$. Using the fact that $\xi_{a b}$ is a coherent state for the direction $\mathbf{n}$ and spin $1 / 2$, one can solve this equation for the Hermitian matrix $L_{a b}$

$$
L_{a b}=e^{2 r_{a b} K \cdot \mathbf{n}_{a b}}=g\left(\mathbf{n}_{a b}\right)\left(\begin{array}{cc}
e^{r_{a b}} & 0 \\
0 & e^{-r_{a b}}
\end{array}\right) g\left(\mathbf{n}_{a b}\right)^{-1},
$$

where $g\left(\mathbf{n}_{a b}\right)$ is a unitary matrix. It is then possible to relate the rapidity $r_{a b}$ to the dihedral angle associated to the triangle $(a b)$.

Let $F_{a}, F_{b}$ be the two future pointing normals determining the two hyperplanes intersecting along the triangle $(a b)$. This triangle is a thick wedge in the terminology of [10] which implies that the corresponding dihedral angle is defined, up to a sign, by

$$
\cosh \Theta_{a b}:=-F_{a} \cdot F_{b}=\frac{1}{2} \operatorname{tr} L_{a b} .
$$

This leads immediately to the identification

$$
\left|r_{a b}\right|=\left|\Theta_{a b}\right|
$$


The sign ambiguity can be related to the $\mu$ parameter of equation (3.10) and, with the canonical choice of phase for the boundary state, one can show that the action at the critical points is given by (4.1) with

$$
\ln \frac{\left\|Z_{b a}\right\|^{2}}{\left\|Z_{a b}\right\|^{2}}=\mu \Theta_{a b}, \quad \text { and } \quad \theta_{a b}=0, \pi
$$

Therefore the action on the critical points corresponding to a non-degenerate 4-simplex yields the Regge action for the Lorentzian 4-simplex determined by the boundary data, up to a term, $c$, that exponentiates to a sign

$$
S=i \mu \gamma \sum_{a<b} k_{a b} \Theta_{a b}+c .
$$

\subsection{Asymptotic formula}

If the boundary data is that of a geometric 4-simplex in Minkowski space, there are two solutions to the critical point equations corresponding to two parity related 4-simplexes. Consequently, the asymptotic formula has two terms. If the boundary state is chosen to be a geometric state, the formula is given, up to a global sign, by the expression

$$
A_{\sigma}(\lambda k, \mathbf{n}) \sim\left(\frac{1}{\lambda}\right)^{12}\left[N_{+} \exp \left(i \lambda \gamma \sum_{a<b} k_{a b} \Theta_{a b}\right)+N_{-} \exp \left(-i \lambda \gamma \sum_{a<b} k_{a b} \Theta_{a b}\right)\right],
$$

where $N_{ \pm}$are constants that do not scale.

We have restricted to Lorentzian boundaries in this paper. It is however important to note that for boundary data corresponding to an Euclidean geometric 4-simplex, the amplitude is not suppressed. We refer the reader to the original article [3] for further details.

\section{Conclusion}

We conclude by summarising our results. In this paper, we have presented results on the asymptotic behaviour of the four-simplex amplitude for the Lorentzian EPRL model. We used stationary phase methods applied to an integral formulation of the amplitude expressed in the coherent state basis. We have calculated the critical point equations and studied their geometry by using the relation between spinors and null vectors in Minkowski space. We have found that the action for the asymptotic problem evaluated on the solutions to the critical point equations yields the Regge action if the boundary data is that of a geometric Lorentzian 4-simplex. In this case, an asymptotic formula was derived.

\section{Acknowledgments}

We would like to thank the organisers of 3rd Quantum Gravity and Quantum Geometry School (Zakopane, February 28 - March 13, 2011) where these results were presented. WF acknowledges support from the Royal Commission for the Exhibition of 1851 and from the Emmy Noether grant ME 3425/1-1 of the German Research foundation (DFG). 


\section{References}

[1] G. Ponzano, T. Regge, "Semiclassical limit of Racah coefficients", Spectroscopic and group theoretical methods in physics, North-Holland Publ. (1968)

[2] J. Engle, E. Livine, R. Pereira and C. Rovelli, "LQG vertex with finite Immirzi parameter," Nucl. Phys. B 799, 136 (2008) [arXiv:0711.0146 [gr-qc]]

[3] J. W. Barrett, R. J. Dowdall, W. J. Fairbairn, F. Hellmann and R. Pereira, "Lorentzian spin foam amplitudes: graphical calculus and asymptotics," Class. Quant. Grav. 27 (2010) 165009 [arXiv:0907.2440 [gr-qc]]

[4] A. Barbieri, "Quantum tetrahedra and simplicial spin networks”, Nucl.Phys. B 518:714-728 (1998) [arxiv:gr-qc/9707010]

[5] J. C. Baez, J. W. Barrett, "The Quantum tetrahedron in three-dimensions and four-dimensions", Adv.Theor.Math.Phys. 3:815-850 (1999) [arxiv:gr-qc/9903060]

[6] E. R. Livine and S. Speziale, “A new spinfoam vertex for quantum gravity”, Phys. Rev. D76:084028 (2007) [arXiv:0705.0674]

[7] J. W. Barrett, R. J. Dowdall, W. J. Fairbairn, H. Gomes and F. Hellmann, "Asymptotic analysis of the EPRL four-simplex amplitude”, J. Math. Phys. 50, 112504 (2009), [arXiv:0902.1170]

[8] J. W. Barrett and L. Crane, “A Lorentzian signature model for quantum general relativity”, Class. Quant. Grav. 17, 3101 (2000) [arXiv:gr-qc/9904025]

[9] J. W. Barrett and L. Crane, "Relativistic spin networks and quantum gravity”, J. Math. Phys. 39, 3296-3302 (1998) [arXiv:gr-qc/9709028]

[10] J. W. Barrett and T. J. Foxon, “Semiclassical limits of simplicial quantum gravity”, Class. Quant. Grav. 11, 543-556 (1994) [arXiv:gr-qc/9310016]. 